WORLD VIEW

\title{
Onchocerciasis: a potential risk factor for glaucoma
}

\author{
P R Egbert, D W Jacobson, S Fiadoyor, P Dadzie, K D Ellingson
}

Series editors: W V Good

and S Ruit

See end of article for authors' affiliations

.....................

Correspondence to:

Peter Egbert, MD,

Department of

Ophthalmology, Stanford

University School of

Medicine, Stanford Eye

Center, 900 Blake Wilbur

Drive, RoomW3002,

Stanford, CA 94305, USA;

egbert@stanford.edu

Accepted for publication

1 January 2005
Br J Ophthalmol 2005;89:796-798. doi: 10.1136/bjo.2004.061895

Background: Onchocerciasis is a microfilarial disease that causes ocular disease and blindness. Previous evidence of an association between onchocerciasis and glaucoma has been mixed. This study aims to further investigate the association between onchocerciasis and glaucoma.

Methods: All subjects were patients at the Bishop John Ackon Christian Eye Centre in Ghana, west Africa, undergoing either trabeculectomy for advanced glaucoma or extracapsular extraction for cataracts, who also had a skin snip biopsy for onchocerciasis. A cross sectional case-control study was performed to assess the difference in onchocerciasis prevalence between the two study groups.

Results: The prevalence of onchocerciasis was $10.6 \%$ in those with glaucoma compared with $2.6 \%$ in those with cataracts (OR, $4.45(95 \% \mathrm{Cl} 1.48$ to 13.43)). The mean age in the glaucoma group was significantly younger than in the cataract group (59 and 65, respectively). The groups were not significantly different with respect to sex or region of residence. In models adjusted for age, region, and sex, subjects with glaucoma had over three times the odds of testing positive for onchocerciasis (OR, 3.50 (95\% $\mathrm{Cl} 1.10$ to 11.18)).

Conclusions: This study has shown a positive association between subclinical onchocerciasis and glaucoma. This finding emphasises the importance of eradication of onchocerciasis from west Africa.
A n estimated 18 million people worldwide are currently infected with onchocerciasis. Onchocerciasis is found in tropical regions, with over $99 \%$ of symptomatic cases occurring in sub-Saharan Africa, and causes blindness and visual impairment in one million to two million individuals. Onchocerciasis is caused by the filarial nematode Onchocerca volvulus, which is transmitted by blackflies in the Simulium damnosum species complex. Infected people may be asymptomatic or have a pruritic skin rash, subcutaneous nodules, lymphadenopathy, or eye disease. However, little is known about the role of onchocerciasis infection in chronic progressive diseases of the eye such as glaucoma.

Ocular onchocerciasis results from an inflammatory reaction around the microfilariae. This initial punctate keratitis is reversible with treatment. Long term infection results in sclerosing keratitis and inflammation in the anterior chamber and retinal epithelium. Posterior involvement may follow, including chorioretinitis, optic neuritis, and optic atrophy. Following years of exposure to the microfilariae, blindness may result. ${ }^{3}$

In west Africa, current efforts to control onchocerciasis include the African Programme for Onchocerciasis Control, using a combined strategy of vector control and chemotherapy with ivermectin. Ivermectin has been shown to reduce or eliminate microfilariae from the eye without significant adverse reaction. ${ }^{45}$ In spite of recent advances in prevention and treatment of onchocerciasis this disease continues as a significant health problem in many areas of west Africa. Until onchocerciasis is eradicated worldwide, a greater understanding of this disease and its manifestations is essential.

Clinically, onchocerciasis produces a low grade inflammation of the eye with formation of peripheral anterior synechiae. ${ }^{67}$ Intraocular pressure can remain normal or even decrease in ocular onchocerciasis. ${ }^{8}$ Pathology in infected eyes shows that although the trabecular system appears unchanged by onchocerciasis when viewed by light microscopy, the post-trabecular outflow system is affected in subjects with both glaucoma and onchocerciasis infection. There is infiltration of inflammatory cells around Schlemm's canal, the efferent veins, the episclera, and the vessels in
Tenon's capsule. This inflammation could possibly raise resistance to outflow of aqueous humour. The presence of microfilariae in Tenon's capsule serves as compelling evidence for onchocerciasis as the cause for the posttrabecular infiltrate. Furthermore, microfilariae have been found in the optic nerve sheath..$^{9-11}$ An older study found an "unexpectedly high prevalence of glaucoma" among patients with onchocerciasis, but the study did not contain control subjects and, therefore, could not calculate an odds ratio or control for other demographic factors to isolate the association. Additionally, the same study found that younger people with glaucoma were more likely to be infected with onchocerciasis. ${ }^{12}$ Other studies also implicate onchocerciasis as a risk factor for optic nerve disease. ${ }^{13}{ }^{14}$ There is more glaucoma in advanced onchocerciasis where eyes tend to have uveitis, peripheral anterior synechiae, and angle closure, but not in those with mild onchocerciasis. ${ }^{8}$ Despite a certain amount of anecdotal, epidemiological, clinical, and pathologic data, there exists only a loose association between onchocerciasis and glaucoma.

Our goal is to contribute to the existing evidence of an association between onchocerciasis and glaucoma in a cross section of patients. In this cross sectional case-control study, we therefore investigated the prevalence of onchocerciasis in patients with glaucoma compared to a control group.

\section{METHODS}

Subjects were patients of the Bishop John Ackon Christian Eye Centre in Cape Coast, Ghana, who had advanced glaucoma and underwent a trabeculectomy or had cataracts and underwent an extracapsular cataract extraction. The cataract patients serve as controls. This study was approved by the investigational review board of the Bishop John Ackon Christian Eye Centre in Cape Coast, Ghana. Records of consecutive patients who underwent either of the above procedures and who also had a skin snip biopsy evaluation for onchocerciasis were evaluated. The skin snip biopsy is standard protocol in the preoperative evaluation for all patients undergoing surgery at this clinic. The laboratory that performed the interpretation was unaware of the 


\begin{tabular}{|ll|}
\hline $\begin{array}{l}\text { Table } 1 \text { Demographic characteristics of the study } \\
\text { population }\end{array}$ \\
\hline Characteristic & No (\%) \\
\hline Mean age & $63.2($ SD 13.6) \\
Sex & $138(48.3)$ \\
Males & $148(51.7)$ \\
Females & \\
Ocular diagnosis & $94(67.1)$ \\
Glaucoma & $192(32.9)$ \\
Cataract & \\
Onchocerciasis & $15(5.2)$ \\
Positive & $271(94.8)$ \\
Negative & $136(48.6)$ \\
Region & $106(37.9)$ \\
Central & $17(6.1)$ \\
Other & $10(3.6)$ \\
Western & $10(3.6)$ \\
Accra & $1(0.2)$ \\
Eastern & 6 \\
Northern & \\
Brong Ahafo & \\
Unspecified & \\
\hline &
\end{tabular}

subjects' ocular diagnosis. Patients under the age of 20 were excluded from the study. Our final sample comprised 286 patients; Table 1 shows their demographic characteristics.

Each patient had a detailed eye examination by an ophthalmologist including visual acuity, slit lamp examination, intraocular pressure, and funduscopic examination. Glaucoma was diagnosed by glaucomatous optic nerve cupping and intraocular pressure greater than $25 \mathrm{~mm} \mathrm{Hg}$. Most patients had very advanced glaucoma with loss of central vision.

We used bivariate analysis to compare the prevalence of onchocerciasis in glaucoma subjects to that of cataract subjects. Further bivariate analyses investigated differences in cataract and glaucoma groups according to age, sex, and region of residence. $\chi^{2}$ Analyses were used for categorical variables and $t$ tests were used for continuous variables. Odds ratios and $95 \%$ confidence intervals were calculated. For the purposes of statistical analysis, region of residence was recoded as a dichotomous variable denoting whether a subject was from the Central Region or from any other region. Finally, to assess the validity of the relation between onchocerciasis and glaucoma we performed multivariate analysis with logistic regression to control for potential confounders. All analyses were conducted in SAS version 8.0 (SAS Institute Inc, Cary, NC, USA).

\section{RESULTS}

The prevalence of onchocerciasis was $10.6 \%$ in those with glaucoma compared with $2.6 \%$ in those with cataracts (table 2). In bivariate analysis, those people with glaucoma had significantly higher odds of onchocerciasis than people with cataracts (OR 4.45 ; 95\% CI 1.48 to 13.43 ).

Further bivariate analyses revealed that there were more women in the cataract group than in the glaucoma group, but the difference was not statistically significant. There was no difference in ethnicity between the two groups; all subjects were black Africans. A $t$ test comparing the average age of the two groups resulted in a significant difference. The glaucoma group was younger (mean age 59 years, SD 15) than the cataract controls (mean age 65 years, SD 13) with a mean difference of 6 years $(\mathrm{p}$ value $=0.0003)$.

After adjusting for potential confounders, (age, sex, region of residence) in a multivariate logistic model, people with glaucoma remained more than three times more likely to test positive for onchocerciasis than people with cataracts (adjusted OR 3.50; 95\% CI 1.10 to 11.18). Age remained significant in the model, but sex and region of residence remained statistically insignificant in the adjusted model (table 3).

\section{DISCUSSION}

In this study, we have found a highly significant association between onchocerciasis and glaucoma. Glaucoma and onchocerciasis are both very important health issues in subSaharan Africa. Glaucoma is the second leading cause of blindness, after cataracts, and is a very difficult disease to treat. $^{15}$ Many ophthalmologists in Ghana believe that onchocerciasis is seen relatively frequently in patients with glaucoma, but the evidence for a link between the two diseases has been mixed. To our knowledge, this is the first study to show that onchocerciasis infection, even in the absence of ocular onchocerciasis, is a potential risk factor for the development of glaucoma. This is supported by previous epidemiological, clinical, and pathological data. Particularly, patients with onchocerciasis have more peripheral anterior synechiae and inflammation in the outflow system than control eyes that could cause increased resistance to aqueous humour outflow. ${ }^{67}$ Furthermore, patients with advanced ocular changes from onchocerciasis have an increased prevalence of glaucoma. ${ }^{8}$ Microfilariae have been found in the optic nerve sheath and could conceivably interfere with optic nerve blood profusion, thereby increasing the susceptibility to glaucoma. ${ }^{10}$

The subjects in our study do not have advanced onchocerciasis eye findings; most of the subjects do not have any visible finding of eye infection on detailed ophthalmological examination. The study provides strong evidence that onchocerciasis, even in the visible absence of ocular damage, is associated with glaucoma.

A limitation of our study is that we cannot say definitively that onchocerciasis is a risk factor for glaucoma because the exposure and outcome data were collected simultaneously. A proved temporal relation of infection with onchocerciasis and

\begin{tabular}{|c|c|c|c|c|c|}
\hline Variable & $\begin{array}{l}\text { Glaucoma } \\
(\mathrm{n}=94)\end{array}$ & $\begin{array}{l}\text { Cataract } \\
(n=192)\end{array}$ & $\begin{array}{l}\text { Test } \\
\text { statistic }\end{array}$ & $\mathrm{p}$ Value & $\begin{array}{l}\text { Odds ratio } \\
(95 \% \mathrm{Cl})\end{array}$ \\
\hline Mean age (SD) & $59(15)$ & $65(13)$ & 3.63 & 0.0003 & \\
\hline Sex & & & & & 0.61 \\
\hline Males & $53(56.4 \%)$ & $85(44.3 \%)$ & 3.708 & 0.054 & (0.37 to 1.01 ) \\
\hline Females & $41(43.6 \%)$ & 107 (55.7\%) & & & \\
\hline Onchocerciasis & & & & & 4.45 \\
\hline Positive & $10(10.6 \%)$ & $5(2.6 \%)$ & 8.196 & 0.0042 & (1.48 to 13.43 ) \\
\hline $\begin{array}{l}\text { Negative } \\
\text { Region }\end{array}$ & $84(89.4 \%)$ & & & 0.0821 & 1.57 \\
\hline $\begin{array}{l}\text { Central } \\
\text { Other }\end{array}$ & $\begin{array}{l}50(56.2 \%) \\
39(43.8 \%)\end{array}$ & $\begin{array}{l}86(45.0 \%) \\
105(55.0 \%)\end{array}$ & 3.0235 & & $(0.94$ to 2.60$)$ \\
\hline
\end{tabular}




\begin{tabular}{lll}
\hline Table 3 & Results of logistic regression & \\
\hline Variable & Beta (standard error) & Odds ratio $(95 \% \mathrm{Cl})$ \\
\hline Onchocerciasis & $1.25(0.59)$ & $3.50(1.10$ to 11.18$)$ \\
Age & $-0.04(0.01)$ & $0.96(0.94$ to 0.98$)$ \\
Region of residence & $-0.65(0.28)$ & $1.92(1.11$ to 3.33$)$ \\
Sex & $-0.61(0.27)$ & $0.54(0.32$ to 0.93$)$ \\
\hline
\end{tabular}

development of glaucoma would give stronger evidence of causality. A frequent problem with case-control studies is that the groups compared are often not identical. This difference between groups may lead to results biased by confounding factors. We controlled for differences in common demographic factors through multivariate analysis. In our study, the cataract control group was older than the glaucoma group. However, because the likelihood of onchocerciasis infection increases with age, the older control group is likely to bias the results towards the null-that is, a better age matched control group may strengthen the association we found. Another limitation of our study is that the results may not apply exactly to other geographical areas. The severity of onchocerciasis eye disease varies from area to area. In Africa, for example, blindness is more common in the savannah and woodland areas than in forest areas. This is probably a consequence of different strains of Onchocerca volvulus. ${ }^{16}$

Knowing that onchocerciasis is associated with glaucoma in this population strengthens the argument to eradicate onchocerciasis from the area by continuation of vector control programmes and drug treatment with ivermectin. This is especially important in light of decreased support for onchocerciasis eradication programmes. ${ }^{17}$ The results of this study suggest that the elimination of onchocerciasis might also reduce the incidence of glaucoma in Ghana.

\section{ACKNOWLEDGEMENTS}

We gratefully acknowledge the help of the staff of the Bishop John Ackon Christian Eye Centre. We thank Lydia Hannah Gould for her entomological expertise.

\footnotetext{
Authors' affiliations

P R Egbert, Stanford University School of Medicine, Department of Ophthalmology, Palo Alto, CA, USA

D W Jacobson, K D Ellingson, Yale University School of Medicine, New Haven, CT, USA

S Fiadoyor, P Dadzie, Bishop John Ackon Christian Eye Centre, Cape Coast, Ghana
}

Sponsor: This study was supported in part by the Elizabeth Butterway Fund for ophthalmic research. None of the authors has any competing interests.

\section{REFERENCES}

1 World Health Organization. Onchocerciasis and its control, Report of a WHO Expert Committee on Onchocerciasis Control, 1995:1-104.

2 Newland HS, White AT, Greene BM, et al. Ocular manifestations of onchocerciasis in a rain forest area of west Africa. $\mathrm{Br} J$ Ophthalmol 1991;75:163-9.

3 Burnham G. Onchocerciasis. Lancet 1998;351:1341-6.

4 Dadzie KY, Awadzi K, Bird AC, et al. Ophthalmological results from a placebo controlled comparative 3-dose ivermectin study in the treatment of onchocerciasis. Trop Med Parasitol 1989;40:355-60.

5 Dadzie KY, Remme J, De Sole G. Changes in ocular onchocerciasis after two rounds of community-based ivermectin treatment in a holo-endemic onchocerciasis focus. Trans R Soc Trop Med Hyg 1991;85:267-71.

6 Yang YF, Cousens S, Murdoch IE, et al. Intraocular pressure and gonioscopic findings in rural communities mesoendemic and nonendemic for onchoceriasis, Kaduna State, Nigeria. Eye 2001;15:756-9.

7 Wormald R, Foster A. Clinical and pathological features of chronic glaucoma in north-east Ghana. Eye 1990;4:107-14.

8 Thylefors B, Duppenthaler JL. Epidemiological aspects of intraocular pressure in an onchocerciasis endemic area. Bull World Health Organ 1979;57:963-9.

9 Rodger F. The pathogenesis and pathology of ocular onchocerciasis. Am J Ophthalmol 1960;49:560-594.

10 Paul EV, Zimmerman LE. Some observations on the ocular pathology of onchocerciasis. Hum Pathol 1970;1:581-94.

11 Stilma JS. Onchocerciasis and glaucoma: ophthalmo-pathological aspects of the limbus and Tenon's capsule in 25 surgical patients from Ghana. Doc Ophthalmol 1981;50:327-35.

12 Berghout E. Onchocerciasis and glaucoma in the forest area of Ghana. Trop Geogr Med 1973;25:233-7.

13 Abiose A, Murdoch I, Babalola O, et al. Distribution and aetiology of blindness and visual impairment in mesoendemic onchocercal communities, Kaduna State, Nigeria. Kaduna Collaboration for Research on Onchocerciasis. Br J Ophthalmol 1994;78:8-13.

14 Cousens SN, Yahaya H, Murdoch I, et al. Risk factors for optic nerve disease in communities mesoendemic for savannah onchocerciasis, Kaduna State, Nigeria. Trop Med Int Health 1997;2:89-98.

15 Egbert PR. Glaucoma in west Africa: a neglected problem. Br J Ophthalmol 2002;86:131-2.

16 Anderson J, Fuglsang H. Ocular onchocerciasis. Trop Dis Bull 1977;74:257-72.

17 Stevenson P. Vision is failing for river-blindness control in Ghana. Lancet 1999;354:2143. 